\title{
Hunting for narrow resonances in the dijet mass spectrum at CMS
}

\author{
Magda Diamantopoulou* \\ On behalf of the CMS Collaboration \\ National Kapodistrian University of Athens, Greece \\ E-mail: melpomeni.diamantopoulou@cern.ch
}

Results from the Dijet Resonance Search using data from 2016 data running of the LHC collected with the CMS detector, with integrated luminosity $36 \mathrm{fb}^{-1}$ will be shown, with emphasis on narrow resonances, for resonance masses above $1.6 \mathrm{TeV}$ and a variety of new physics models used for the interpretation of the experimental results. The traditional method of estimating the QCD background is used, employing a parametrization for the background and an empirical fit of the data in the signal region.

Sixth Annual Conference on Large Hadron Collider Physics (LHCP2018)

4-9 June 2018

Bologna, Italy

\footnotetext{
* Speaker.
} 


\section{Introduction}

The Standard Model (SM) of particle physics is the theory which describes electromagnetic, weak and strong interactions between quarks and leptons. However, the Standard Model still cannot explain key concepts in our understanding of the universe. For instance, why is there an imbalance of matter and antimatter in the universe? How does gravity fit into our model? Why are there exactly three families of fundamental particles? Models of physics, that extend the standard model (SM) often require new particles that couple to quarks and/or gluons and decay to dijets. The natural width of resonances in the dijet mass (mjj) spectrum increases with the coupling, and may be either narrow or broad compared to the experimental resolution [1].

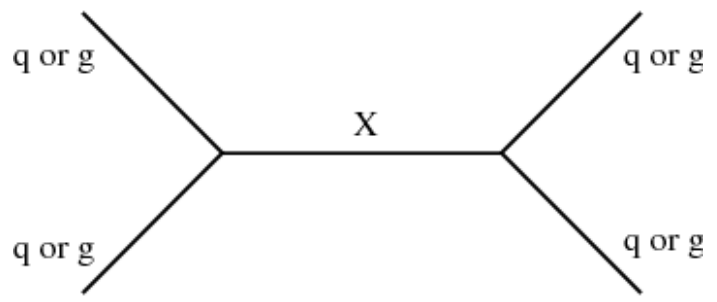

Figure 1: Pseudo-Feynman Diagram of dijet resonance. The initial state and final state both contain two partons (quarks, antiquarks or gluons) and the intermediate state contains an s-channel resonance X.

The dijet system is typically composed of the two jets with the highest transverse momentum $\left(p_{T}\right)$ in an event and the dijet mass is given by the equation: $m_{j j}=\sqrt{\left(E_{j 1}+E_{j 2}\right)^{2}-\left(\vec{p}_{j 1}+\vec{p}_{j 2}\right)^{2}}$, where $j 1$ and $j 2$ denote the leading (highest in $p_{T}$ ) and sub-leading (second highest in $p_{T}$ ) jets. The data used in the analysis were collected with the CMS experiment in 2016, from pp collisions at the LHC at a $\sqrt{s}=13 \mathrm{TeV}$ corresponding to an integrated luminosity of the data is $36 \mathrm{fb}^{-1}$. The results were published in [2] and similar searches have been published previously by the ATLAS and CMS Collaborations at $\sqrt{s}=13 \mathrm{TeV}$ (see [3], [4], [5], [6]).

\section{CMS Experiment at LHC}

The CMS detector [7] consists of a superconducting solenoid which provides an axial field of 3.8 T. Inside the solenoid volume are located the silicon pixel and strip tracker $(|\eta|<2.4)$ and the barrel and endcap calorimeters $(|\eta|<3.0)$. Outside the solenoid volume $(3.0<|\eta|<5.0)$, a steel and quartz-fiber hadron forward calorimeter is located.

\section{Jet Reconstruction and Event Selection}

A particle-flow (PF) event algorithm is used to reconstruct and identify each individual particle with an optimized combination of information from the various elements of the CMS detector. Jets are reconstructed with the anti- $k_{T}$ clustering algorithm [8], [9] applied to the particles resulting from the PF algorithm [10] with a distance parameter of 0.4, as implemented in the FASTJET package [11]. Spatially close jets are combined into "wide jets" and used to determine the dijet mass. The dijet selection requires that the distance in pseudorapidity between the two jets is $|\Delta \eta|<$ 1.3 in order to suppress the QCD (t-channel) and enhance the signal (s-channel), and the dijet mass to be greater than $1246 \mathrm{GeV}$, which is the threshold above which the trigger is $>99 \%$ efficient. 


\section{Dijet Data Quality and Narrow Resonance Search}

We perform comparisons of the data to Pythia [12] QCD background predictions for the dijet events, to check that the data are of high quality. Fig. 2 (right) shows the $\Delta \phi$ distribution between the two leading wide-jets with a peak at $\pi$ as expected from QCD dijet production. Fig. 2 (left) also shows the dijet $|\Delta \eta|$ distribution, where $\eta$ is the pseudorapidity, dominated by t-channel parton exchange as expected from QCD, which increases with increasing $|\Delta \eta|$.
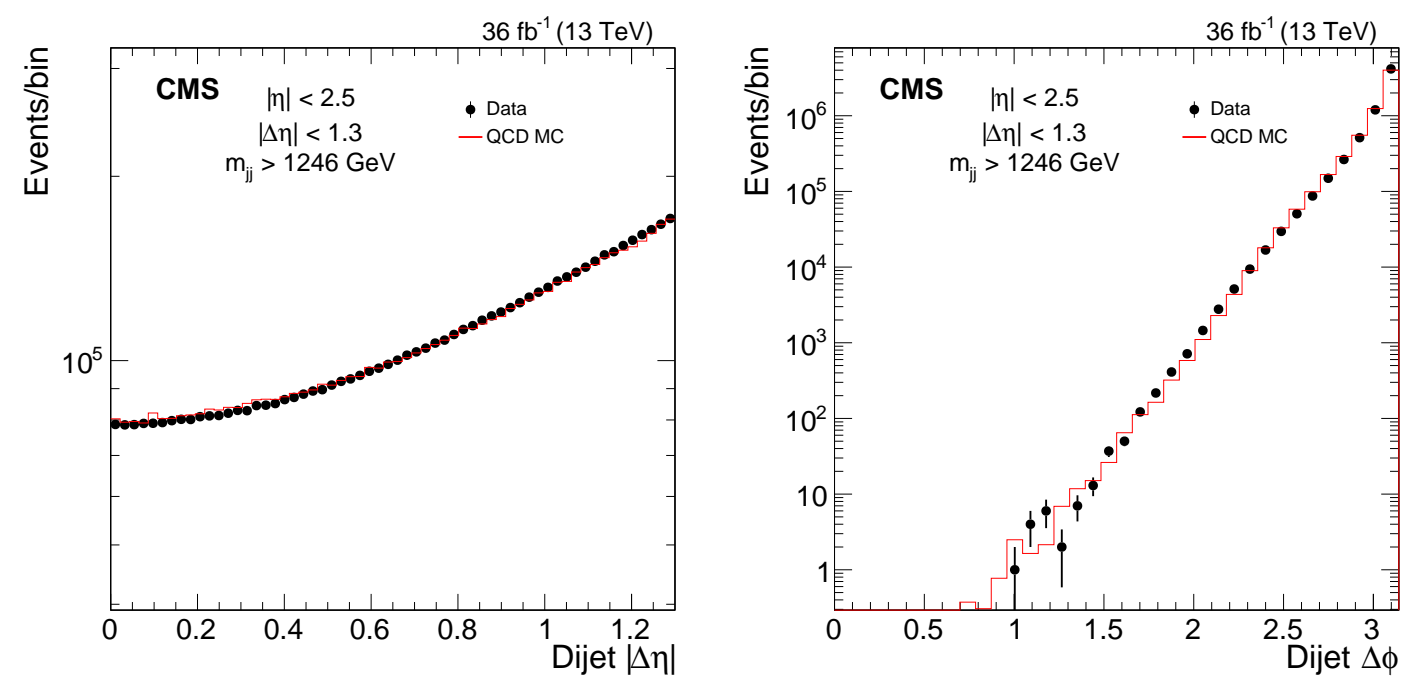

Figure 2: The pseudorapidity (left) and the azimuthial angular seperation (right) between two wide jets. Data are shown (black points) along with Pythia simulation (red line) [2].

A fit with an empirical parametrization is performed to the data and its parameters are treated as unconstrained nuisance parameters in the hypothesis testing:

$$
\frac{d \sigma}{d m_{j j}}=\frac{P_{0}(1-x)^{P_{1}}}{x^{P_{2}+P_{3} \ln (x)}}
$$

where $x=m_{j j} / \sqrt{s}$ and $P_{0}, P_{1}, P_{2}$, and $P_{3}$ are four free fit parameters. The chi-squared per number of degrees of freedom of the fit is $\chi^{2} / \mathrm{NDF}=38.9 / 39$.

There is no evidence for a narrow resonance, as indicated from Fig. 3 showing the dijet mass spectrum, along with a background only fit (left). We therefore proceed with the extraction of cross section limits shown in Fig. 3 (right) and mass limits presented in Table 1. 

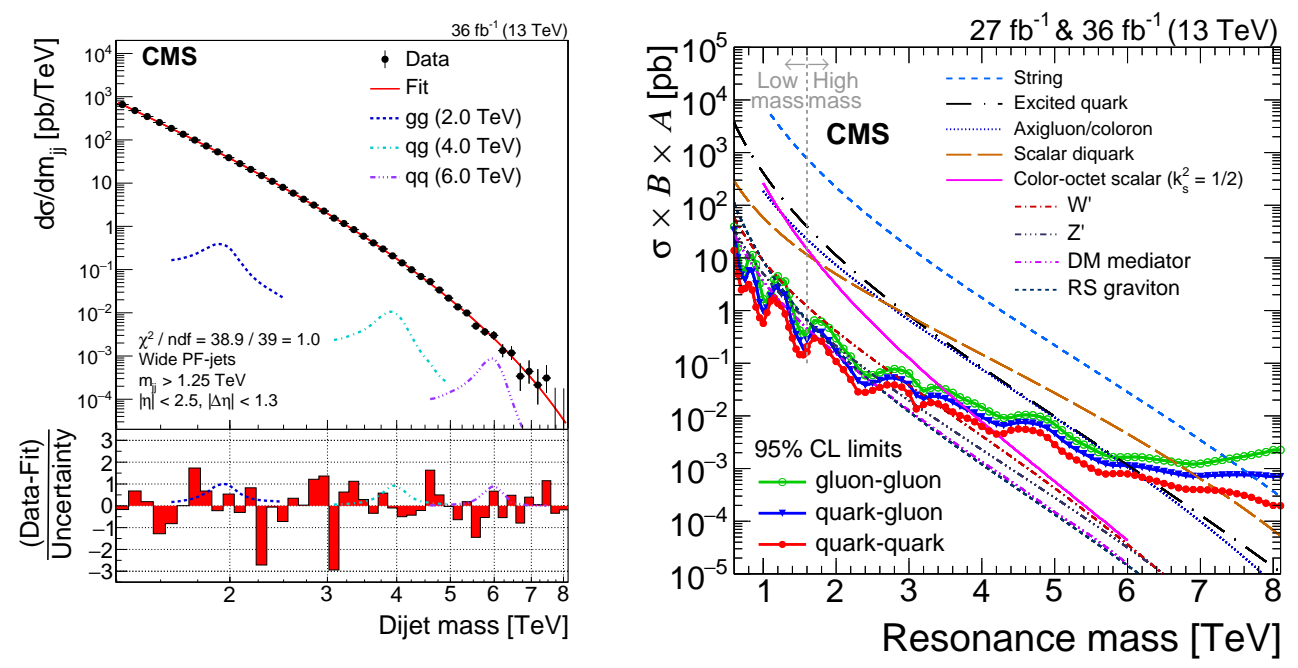

Figure 3: The data Dijet Mass spectrum (black points) compared to a fitted parameterization of the background (solid curve) on top, and the difference between the data and the fitted parametrization, divided by the statistical uncertainty of the data on bottom (left) and the observed 95\% CL upper limits on the product of the cross section, branching fraction, and acceptance for quark-quark, quark-gluon, and gluon-gluon dijet resonances. Limits are compared to the predicted cross sections for string resonances, excited quarks, axigluons, colorons, scalar diquarks, color-octet scalars, new gauge bosons $\mathrm{W}$ and $\mathrm{Z}$ with SM-like couplings, dark matter mediators for $\mathrm{m} \mathrm{DM}=1 \mathrm{GeV}$, and $\mathrm{RS}$ gravitons (right) [2].

Table 1: Observed and expected mass limits at 95\% CL from this analysis compared to previously published limits on narrow resonances from CMS with $12.9 \mathrm{fb}^{-1}$.

\begin{tabular}{lccc}
\hline Model & $\begin{array}{c}\text { Final } \\
\text { State }\end{array}$ & $\begin{array}{c}\text { Observed (expected) } \\
36 \mathrm{fb}^{-1}\end{array}$ & $\begin{array}{c}\text { mass limit }[\mathrm{TeV}] \\
12.9 \mathrm{fb}^{-1}\end{array}$ \\
\hline String resonance & $\mathrm{qg}$ & $7.7(7.7)$ & $7.4(7.4)$ \\
Scalar diquark & $\mathrm{qq}$ & $7.2(7.4)$ & $6.9(6.8)$ \\
Axigluon/coloron & $\mathrm{q} \bar{q}$ & $6.1(6.0)$ & $5.5(5.6)$ \\
Excited quark & $\mathrm{qg}$ & $6.0(5.8)$ & $5.4(5.4)$ \\
Color-octet scalar $\left(k_{s}^{2}=1 / 2\right)$ & $\mathrm{gg}$ & $3.4(3.6)$ & $3.0(3.3)$ \\
W' SM-like & $\mathrm{q} \bar{q}$ & $3.3(3.6)$ & $2.7(3.1)$ \\
Z' SM-like & $\mathrm{q} \bar{q}$ & $2.7(2.9)$ & $2.1(2.3)$ \\
RS graviton $\left(k / \bar{M}_{P l}=0.1\right)$ & $\mathrm{q} \bar{q}, \mathrm{gg}$ & $1.8(2.3)$ & $1.9(1.8)$ \\
DM mediator $\left(m_{D M}=1 \mathrm{GeV}\right)$ & $\mathrm{q} \bar{q}$ & $2.6(2.5)$ & $2.0(2.0)$ \\
\hline
\end{tabular}




\section{Conclusions}

The narrow resonance search using data collected in 2016 with the CMS experiment and corresponding to an integrated luminosity $36 \mathrm{fb}^{-1}$ extends the limits previously reported by CMS in the dijet channel, resulting in the most stringent constraints on many of the models considered.

\section{References}

[1] R. M. Harris and K. Kousouris, Searches for dijet resonances at hadron colliders, Int. J. Mod. Phys. A 26 (2011) 5005, doi:10.1142/S0217751X11054905, arXiv:1110.5302.

[2] CMS Collaboration, Search for narrow and broad dijet resonances in proton-proton collisions at $\sqrt{s}=$ $13 \mathrm{TeV}$ and constraints on dark matter mediators and other new particles, JHEP 08 (2018) 130, arXiv:arXiv:1806.00843

[3] ATLAS Collaboration, Search for low-mass dijet resonances using trigger-level jets with the ATLAS detector in pp collisions at $\sqrt{s}=13$ TeV Phys. Rev. Lett. 121, 081801 (2018), arXiv:1804.03496

[4] CMS Collaboration, Search for dijet resonances in proton-proton collisions at $\sqrt{s}=13 \mathrm{TeV}$ and constraints on dark matter and other models, Phys. Lett. B 769 (2017) 520, doi:10.1016/j.physletb.2017.02.012, arXiv:1611.03568.

[5] CMS Collaboration, Search for narrow resonances decaying to dijets in proton-proton collisions at $\sqrt{s}$ = 13 TeV, Phys. Rev. Lett. 116 (2016) 071801, doi:10.1103/PhysRevLett.116.071801, arXiv:1512.01224.

[6] ATLAS Collaboration, Search for new phenomena in dijet mass and angular distributions from pp collisions at $\sqrt{s}=13 \mathrm{TeV}$ with the ATLAS detector, Phys. Lett. B 754 (2016) 302, doi:10.1016/j.physletb.2016.01.032, arXiv:1512.01530.

[7] CMS Collaboration, The CMS experiment at the CERN LHC, JINST 3 (2008) S08004, doi:10.1088/1748-0221/3/08/S08004

[8] M. Cacciari and G. P. Salam, Dispelling the $N^{3}$ myth for the $k_{t}$ jet-finder, Phys. Lett. B 641 (2006) 57, doi:10.1016/j.physletb.2006.08.037, arXiv:hep-ph/0512210.

[9] M. Cacciari, G. P. Salam, and G. Soyez, The anti- $k_{t}$ jet clustering algorithm, JHEP 04 (2008) 063, doi:10.1088/1126-6708/2008/04/063, arXiv:0802.1189.

[10] CMS Collaboration, Particle-flow reconstruction and global event description with the CMS detector, JINST 12 (2017) P10003, doi:10.1088/1748-0221/12/10/P10003, arXiv:1706.04965.

[11] M. Cacciari, G. P. Salam, and G. Soyez, FastJet user manual, Eur. Phys. J. C 72 (2012) 1896, doi:10.1140/epjc/s10052-012-1896-2, arXiv:1111.6097.

[12] T. Sjöstrand, S. Mrenna, and P. Skands, A brief introduction to PYTHIA 8.1, Comp. Phys. Comm. 178 (2008) 852, doi:10.1016/j.cpc.2008.01.036, arXiv:0710.3820. 